\title{
Micromagnetic Simulation of Thermal Effects in Magnetic Nanostructures
}

\author{
Rok Dittrich, Thomas Schrefl, Vassilios Tsiantos, Hermann Forster, Dieter Suess, Werner \\ Scholz, and Josef Fidler \\ Solid State Physics, Vienna University of Technology, \\ Wiedner Haupstr. 8-10/138, A-1040 Vienna, Austria
}

\begin{abstract}
A path finding method and a stochastic time integration scheme for the simulation of thermally activated magnetization processes are introduced. The minimum energy path and the saddle points for the thermally induced transitions between the ground states of NiFe magnetic nanoelements are calculated.
\end{abstract}

\section{INTRODUCTION}

With decreasing size of magnetic nanostructures thermal effects become increasingly important. Prominent examples are magnetization noise in magnetic sensor elements [1-3] and the thermal stability of magnetic MRAM (Magnetic Random Access Memory) cells [4] or magnetic storage media [5]. Magnetic sensors require a high sensitivity so that small magnetic fields can be detected. On the other hand thermal fluctuations which will lead to thermal noise should be suppressed as good as possible. The free layer of a multiplayer sensor element is soft magnetic hand may have a size well below one micrometer. Thermally induced magnetization processes may cause local or global magnetization rotations which cause the magnetization noise. With decreasing lateral extension of the elements the energy barrier which hinders spontaneous changes of the magnetization decreases. Magnetic storage elements require a low and well defined switching field which in practice is limited by the current through the write line in an array of MRAM cells. On the other hand the shape or the induced anisotropy should guarantee a life time of a stored bit of about 10 years. Again the energy barrier for thermally induced magnetization reversal decreases with increasing size of the storage elements. The corresponding time scales differs by several orders of magnitude: Thermal noise arises on a time scale of a few nanoseconds; thermally induced switching of the magnetization over energy barriers extends over seconds to years. Random thermal fluctuations of the magnetization are the underlying physical process which cause both thermal noise and spontaneous switching. The stochastic fluctuations arise from the interplay between the lattice vibrations and the magnetization.

A micromagnetic system will be close to a local minimum the total magnetic Gibb's free energy. Thermal fluctuations of the magnetization cause the magnetization to wander around near this minimum. Occasionally the system will reach a region next to a saddle point. The system may cross the energy barrier and move into the basin of attraction of a different energy minimum. This process can be described by the Neel-Brown theory [6,7]. The relaxation time, $\tau=f_{0}^{-1} \exp \left(E_{\mathrm{b}} / k_{\mathrm{B}} T\right)$, is the inverse of probability per unit time for crossing the barrier $E_{\mathrm{b}}$. The attempt frequency, $f_{0}$, depends on material parameters, like anisotropy, particle shape, and damping [8]. Its value, which ranges from $f_{0}=10^{9} \mathrm{~Hz}$ to $f_{0}=10^{12} \mathrm{~Hz}$, sets the time scale for thermally assisted magnetization reversal, $\tau_{0}=f_{0}^{-1} \approx 1 \mathrm{~ns}$. 
The theoretical treatment of thermally induced magnetization processes starts from the stochastic Landau-Lifshitz Gilbert equation and the corresponding Fokker-Planck equation [7]. The energy barrier can be calculated for coherent rotation in single domain particles and the formation of reversed domains in thin ferromagnetic wires. The attempt frequency can be estimated solving the Fokker-Planck equation numerically or analytically [7-10]. Alternatively, the stochastic Landau-Lifshitz Gilbert equation can be solved numerically for short time scales and small systems [11-14]. Recently, numerical solutions of the stochastic Landau-Lifshitz Gilbert equation were reported for extended micromagnetic systems [3,14]. Zhu [3] analyzed the magnetization noise in submicron sized sensor elements based on the numerical solution of the stochastic Landau-Lifshitz Gilbert equation. The time integration of the stochatistic LandauLifshitz equation is restricted to small time scales and thus is proper tool to analyze magnetization noise. The calculation of long term thermal effects needs a detailed characterization of the energy landscape along the most probable path which is taken by the system to cross the energy barrier. Berkov [15] calculated the transition path of interacting single domain particles, minimizing the action along the path. He showed that a direct minimization of the action may also give paths through local maxima which have to be excluded. Ren [16] proposed an elastic band method to calculate a minimum path in micromagnetic systems. Starting from an initial guess for the path which connects two local minima of the system, a highly probable path is found moving the points along the path according to an algorithm which resembles tensioning an elastic band across a mountain. Varients of elastic band methods methods are commonly used to calculate transition rates in physical chemistry [17]. Dittrich an co-workers [19] originally applied the elastic band method to calculate energy barriers and saddle points in complex micromagnetic systems like discrete perpenpendicular recording media.

In this work we apply both stochastic time integration and path finding techniques in the framework of the finite element method, in order to simulate thermal effects in magnetic nanostructures. Thus it is possible to take into account complex geometries and realistic element shapes. Both methods are complementary. The stochastic time integration is restricted to simulation times of about $10 \mathrm{~ns}$. As a consequence the calculation of barrier crossing by stochastic time integration is limited to small energy barriers. The transition rate for large barriers can be estimated fromthe barrier height which can be calculated from the minimum energy path. In addition to the energy barrier, the elastic band method provides a global view of the energy landscape such as local minima and saddle point along the path. The magnetization processes as computed from the stochastic time integration method and the minimum energy path are compared for transitions between different ground states in magnetic nano-elements.

\section{MICROMAGNETIC AND NUMERICAL BACKGROUND}

The micromagnetic description of the system starts from the total magnetic Gibbs' free energy [19]

$$
E=\int\left(A\left[\sum_{i}^{x, y, z}\left(\nabla u_{i}\right)^{2}\right]+K_{1}[1-(\mathbf{u} \cdot \mathbf{a})]-\frac{J_{\mathrm{s}}}{2}\left(\mathbf{u} \cdot \mathbf{H}_{\mathrm{s}}\right)-J_{\mathrm{s}}\left(\mathbf{u} \cdot \mathbf{H}_{\mathrm{ext}}\right)\right) \mathrm{d} V
$$

$E$ is the sum of the exchange energy, the anisotropy energy, the stray field energy, and the Zeeman energy; $\mathbf{u}$ denotes the unit vector parallel to the magnetization, $A$ is the exchange constant, $K_{1}$ is the uniaxial magnetocrystalline anisotropy constant, and $J_{\mathrm{s}}$ is the spontaneous 
magnetic polarization. The integral (3) is over the total volume of the magnetic particles. In a stationary state the magnetic system occupies a local minimum of (1). Owing to thermal activation the system may overcome an energy barrier and spontaneously move towards a different local minimum of the energy.

We use the finite element method to evaluate $E$ for complex magnetic systems. The direction cosines of the magnetization, $u_{\mathrm{k}}$, are interpolated by piecewise linear functions on a tetrahedral finite element mesh. In order to calculate the magnetic stray field, $\mathbf{H}_{\mathrm{s}}$, we use a hybrid finite element / boundary element method [20]. The simulation of the time evolution of the magnetization requires to calculate the effective field, $\mathbf{H}_{\text {eff }}$, defined by the negative variational derivative of $E$. The effective field on the nodes of the finite element mesh may be approximated using a box scheme

$$
\mathbf{H}_{\text {eff }}^{l}=-\frac{1}{\mu_{0}} \frac{\partial E}{\partial \mathbf{m}^{l}}
$$

where $\mathbf{m}^{l}$ is the magnetic moment associated with node $l$ of the finite element mesh. The stochastic Landau-Lifshitz equation is a system of $3 N$ Langevin equations with multiplicative noise

$$
\begin{aligned}
& \frac{\partial u_{i}^{l}}{\partial t}=A_{i}^{l}(t)+\sum_{k} B_{i k}^{l}(t) H_{\mathrm{th}, k}^{l}(t) \\
& A_{i}^{l}=\left[-\frac{|\gamma|}{1+\alpha^{2}}\left(\mathbf{u}^{l} \times \mathbf{H}_{\text {eff }}^{l}\right)-\frac{|\gamma| \alpha}{1+\alpha^{2}}\left(\mathbf{u}^{l} \times\left(\mathbf{u}^{l} \times \mathbf{H}_{e f f}^{l}\right)\right)\right]_{i} \\
& B_{i k}^{l}=-\gamma \mid \sum_{j} \varepsilon_{i j k} u_{j}^{l}-\frac{|\gamma| \alpha}{1+\alpha^{2}}\left(u_{i}^{l} u_{k}^{l}-\delta_{i k}\right)
\end{aligned}
$$

where the indices $i, j, k$ run over the three space directions, and the index $l=1, \ldots, N$ runs over the number of nodes. The drift term, $A_{i}^{l}$, is the right hand side of the deterministic Landau-LifshitzGilbert equation. $\alpha$ is the Gilbert damping constant and $\gamma$ is the gyromagnetic ratio. $\mathbf{H}_{\mathrm{th}}^{l}$ is the random thermal field. The thermal field is assumed to be a Gaussian random process with the following statistical properties:

$$
\left\langle H_{\mathrm{th}, i}^{l}(t)\right\rangle=0 \text { and }\left\langle H_{\mathrm{th}, i}^{l}(t) H_{\mathrm{th}, j}^{k}\left(t^{\prime}\right)\right\rangle=2 D \delta_{i j} \delta_{l k} \delta\left(t-t^{\prime}\right)
$$

The average of the thermal field, take over different realization, vanishes in each direction $i$ in space. The thermal field is uncorrelated in time and space. The strength of the thermal fluctuations follow form the fluctuation-dissipation theorem [11]:

$$
D=\frac{\alpha k_{\mathrm{B}} T}{|\gamma| \mu_{0} \mathbf{m}^{l}}
$$


Numerically the equations (3) to (5) are be solved using semi-implicit time integration method [21]. The time is divided into a lattice of discrete point $t_{\mathrm{n}}$. The Langevin equation is solved in the time interval $t_{n}$ to $t_{n+1}$ with the initial condition $u_{i}^{l}=u_{i}^{l}\left(t_{n}\right)$. The right hand side of (5) is evaluated at the midpoint $\bar{t}_{n}=\left(t_{n}+t_{n+1}\right) / 2=t_{n}+\Delta t / 2$. The update of the direction cosines is given by

$$
\Delta u_{i}^{l}=A_{i}^{l}\left(\ldots, \bar{u}_{i}^{l-1}, \bar{u}_{i}^{l}, \bar{u}_{i}^{l+1}, \ldots\right) \Delta t+\sum_{k} B_{i k}^{l}\left(\ldots, \bar{u}_{i}^{l-1}, \bar{u}_{i}^{l}, \bar{u}_{i}^{l+1}, \ldots\right) \Delta H_{\mathrm{th}, k}^{l}
$$

with the noise integral

$$
\Delta H_{\mathrm{th}, k}^{l}=\int_{t_{n}}^{t_{n+1}} H_{\mathrm{th}, k}^{l}(t) \mathrm{d} t .
$$

The magnetization directions at the midpoint of the time interval is

$$
\bar{u}_{i}^{l}=\left(u_{i}^{l}\left(t_{n}\right)+u_{i}^{l}\left(t_{n+1}\right)\right) / 2=u_{i}^{l}\left(t_{n}\right)+\Delta u / 2,
$$

assuming linearization of $u_{i}^{l}$ within the time interval. The midpoint value, $\bar{u}_{i}^{l}$, is given implicitly by the equations (8) and (10) which is solved by functional iteration. Numerical tests show that about five iterations are sufficient to gain the required accuracy of $10^{-5}$. From the midpoint value the magnetization at the time $t_{n+1}$ is calculated from (10) and then normalized:

$$
\tilde{u}_{i}^{l}\left(t_{n+1}\right)=2 \bar{u}_{i}^{l}-u_{l}^{l}\left(t_{n}\right), \quad \mathbf{u}^{l}=\tilde{\mathbf{u}} /|\tilde{\mathbf{u}}| .
$$

A common feature of path finding methods is the discrete representation of the path connecting the inital state of the system with its final state. In micromagnetics we represent the magnetic states of a system by the set of magnetic moments $\mathbf{M}=\left\{\ldots, \mathbf{m}^{l-1}, \mathbf{m}^{l}, \mathbf{m}^{l+1}, \ldots\right\}$.The index $l=1, \ldots, N$ runs over all nodes of the finite element mesh. For the configuration space we use polar coordinates of dimension $2 N$. First we construct a sequence of magnetic states in such a way as to form a discrete representation of a path from the initial magnetization state, $\mathbf{M}^{(\mathrm{i})}$, to the final magnetization state, $\mathbf{M}^{(\mathrm{f})}$. An optimization algorithm is then applied until at any point along the path the gradient of the energy is only pointing along the path. This path is called minimum energy path which means that the energy is stationary for any degree of freedom perpedicular to the path. The minimum energy path typically represents the path with the greatest statistical weight. From this path statistical quantities as for example transition rates for the thermally induced magnetization reversal can be estimated.

Henkelman and Jónsson proposed the nugded elastic band method to calculate minimum energy paths [17]. We represent a path by a sequence of images. An intitial path is assumed which connects the initial magnetization state $\mathbf{M}^{(\mathrm{i})}=\mathbf{M}^{(1)}$ with the final magnetization state $\mathbf{M}^{(\mathrm{f})}=\mathbf{M}^{(m)}$. The index $k$ runs from 1 to $m$. The path is optimal, if for any image $\mathbf{M}^{(k)}$ the gradient of the energy is only pointing along the path or in other words the component of the energy gradient normal to the path, $\mathbf{D}$, is zero. If $\mathbf{t}$ denotes the unit tangent vector along the path, the optimal path have the following property, a minimum energy path has the following property 


$$
\mathbf{D}^{(k)}=\nabla E\left(\mathbf{M}^{(k)}\right)-\left[\nabla E\left(\mathbf{M}^{(k)}\right) \cdot \mathbf{t}\right] \mathbf{t}=0, \text { for } k=1, \ldots, m
$$

The optimal path can be found using an iterative scheme. In each iteration step the images towards lower energy in a direction perpendicular to the path. So the image $\mathbf{M}^{(k)}$ is moved into the direction, $-\mathbf{D}^{(k)}$. This iterative scheme numerically is very ineffective. Thus instead we solve a system of ordinary differential equations

$$
\frac{\partial \mathbf{M}^{(k)}}{\partial t}=-\mathbf{D}^{(k)} \quad \text { for } k=2, \ldots,(m-1)
$$

using an implicit, variable order, variable time step time integration method [22]. Here the time $t$ is introduced for numerical convenience and has no physical meaning. In keep an equal distance between successive images a spring force may be introduced [17].

\section{RESULTS}

Small rectangular NiFe elements show two distinct ground: The S-state and the C-state. Thermal fluctuations may induce a transition between the different states. The C-state has a slightly lower energy than the S-state. Figure 1 shows the magnetic states during the thermally induced switching from the $\mathrm{S}$-state to the $\mathrm{C}$-state. The magnetization distributions are calculated from the numerical solution of the stochastic Landau-Lifshitz Gilbert equation at a temperature of $T=350 \mathrm{~K}$. The sequence of magnetization configurations in figure 1 covers a time of $5 \mathrm{~ns}$.

The transition between the grounds states were also calculated using the path finding method. The initial path was constructed connecting the two possible S-states. Along the initial path the different images are obtained by linear interpolation. Then a minimum energy path is found solving equation (13). Figure 2 shows the energy along the final path. In addition the magnetization states along the path are shown. The path finding algorithm identifies the C-state as a global energy minimum. The $\mathrm{C}$-state is separated from the $\mathrm{S}$ states by saddle points. The comparison of the magnetization states presented in figure 1 and in figure 2 clearly shows that the magnetic states along the minimum energy path correspond to the magnetic states which are visited during the stochastic motion of the magnetization according to the Langevin equation.

\section{ACKNOWLEDGEMENT}

This work was supported by the Austrian Science Fund (Y-132 PHY).
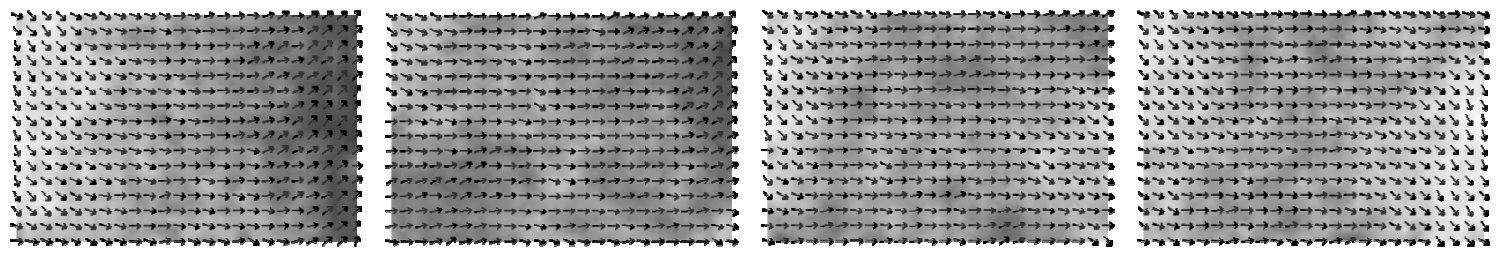

Figure 1. Magnetization states visited during the solution of the stochastic Landau-Lifshitz Gilbert equation. The NiFe element has an extension of $150 \times 100 \times 5 \mathrm{~nm}^{3}$. A induced anisotropy of $K_{1}=500 \mathrm{~J} / \mathrm{m}^{3}$ was assumed parallel to the long axis. The grey scales maps the magnetization component parallel to the short axis of the element. 

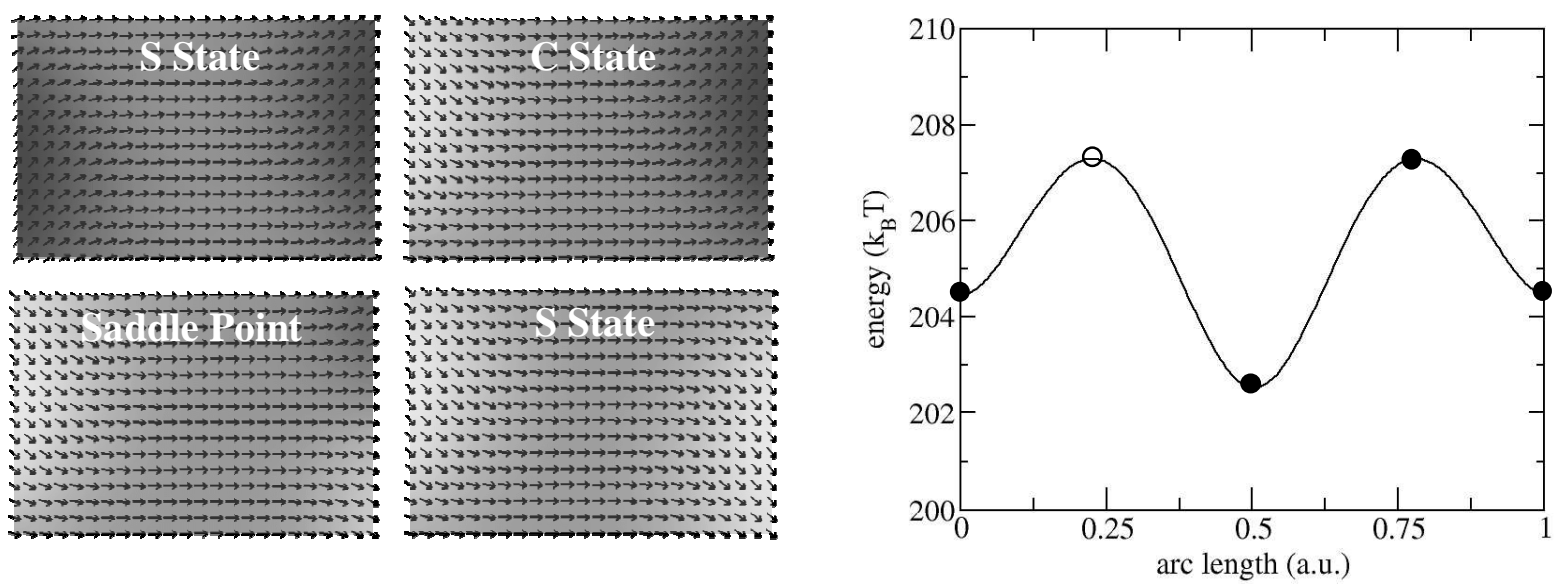

Figure 2. Magnetization configurations and energy along the minimum energy path. The temperature is $T=350 \mathrm{~K}$. The saddle point with the open symbols is not shown on the left hand side.

\section{REFERENCES}

1. N. Smith and P. Arnett, Appl. Phys. Lett. 78, 1448 (2001).

2. H. N. Bertram, V. L. Safonov, Z. Jin, IEEE Trans. Magn. 38, 2514 (2002).

3. J.-G. Zhu, J. Appl. Phys. 91, 7273 (2002).

4. N. D. Rizzo, M. DeHerrera, J. Janesky, B. Engel, J. Slaughter and S. Tehrani, Appl. Phys. Lett. 80, 2335 (2002).

5. D. Weller and A. Moser, IEEE Trans. Magn. 35, 4423 (1999).

6. L. Néel, Ann. Geophys. 5, 99 (1949).

7. W. F. Brown, Jr., Phys. Rev. 130, 1677 (1963).

8. H.-B. Braun, J. Appl. Phys. 76, 6310 (1994).

9. H. Fukushima, Y. Uesaka, Y. Nakatani and N. Hayashi, J. Magn. Magn. Mater. 242-245, 1002 (2002).

10. W. T. Coffey, D. S. F. Crothers, J. L. Dorman, Yu. P. Kalmykov, E. C. Kennedy and W. Wernsdorfer, Phys. Rev. Lett. 80, 5655 (1998).

11. J. L. Garcia-Palacios and F. J. Lazaro, Phys. Rev. B 58, 14937 (1998).

12. K. Zhang, D. R. Fredkin, J. Appl. Phys. 85, 5308 (1999).

13. W. Scholz, T. Schrefl and J. Fidler, J. Magn. Magn. Mater. 233, 296 (2001).

14. W. Scholz, T. Schrefl and J. Fidler, IEEE Trans. Magn. 36, 3189 (2000).

15. D.V. Berkov, J. Magn. Magn. Mater. 186, 199 (1998).

16. Weinan E,W. Ren and E. Vanden-Eijnden, Phys. Rev. B 66, 52301 (2002)

17. G. Henkelman, H. Jónsson, J. Chem. Phys. 113, 9978 (2000).

18. R. Dittrich, T. Schrefl, D. Suess, W. Scholz, H. Forster and J. Fidler, J. Magn. Magn. Mater. 250, 12 (2002).

19. W. F. Brown, Jr, Micromagnetism, (Interscience, New York,1963).

20. D.R. Fredkin, T.R. Koehler, IEEE Trans. Magn. 26, 415 (1990).

21. P. D. Drummond and I. K. Mortimer, J. Comput. Phys. 93, 144 (1991).

22. S. D. Cohen and A. C. Hindmarsh, Computers in Physics 10, 138 (1996). 